(Aus dem physiologischen Institute der k. k. böhmischen Universität in Prag.)

\title{
Ein experimenteller Beitrag zur Lehre von der individuellen Konstanz der Harnsäure beim Menschen.
}

Von

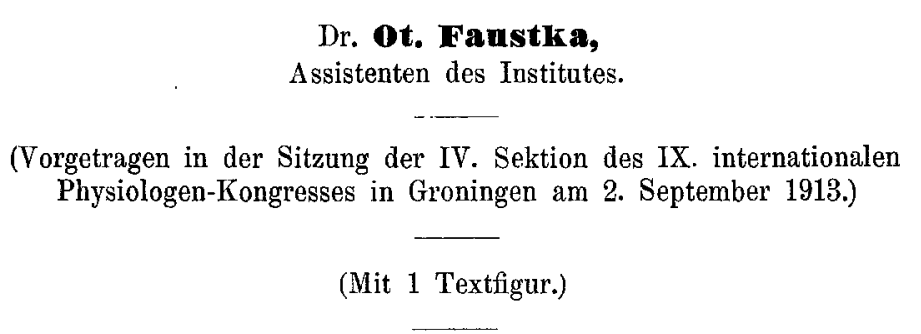

Die individuelle Konstanz der Menge der in einer bestimmten Periode des nüchternen Zustandes ausgeschiedenen Harnsäure beim Menschen hat zuerst Marešl) im Jahre 1887 nachgewiesen. Die Entdeckung dieser Tatsache, welche auf eine individuell charakterisierte Quelle der Harnsäure, das Protoplas ma, hinweist, bildet den ersten tatsächlichen Grund, auf dem Mareš seine Theorie über die Herkunft der Harnsäure beim Menschen aufgebaut hat. Seine damaligen ursprünglichen Versuche bezweckten, die Verhältnisse zwischen der Menge der ausgeschiedenen Harnsäure und der Menge des zu gleicher Zeit ausgeschiedenen Harnstickstoffs aufzuhellen, und haben zuerst den physiologischen Nachweis geliefert, dass die Harnsäureausfuhr beim Menschen ganz andere Verhältnisse zeigt als die des Harnstoffes, so dass diese beiden Körper verschiedenen Ursprungs sein dürften, und zwar so, dass der Harnstoff aus dem verdauten und resorbierten Nabrungseiweiss herstammt, die Harnsäure aber aus stofflichen Änderungen im Zellprotoplasma selbst hervorgeht ${ }^{2}$ ).

In seiner diesbezüglichen grundlegenden Arbeit $^{3}$ ) formuliert Mareš seine Hypothese wie folgt: L'acide urique est le produit

1) F. Marěs, Sur l'origine de l'acide urique chez l'homme. Arch. slaves de biol. t. 3 p. 207-226. 1887. - Sborník lékařský t. 2 p. 1-18. 1888.

2) F. Mare ̌̌, Der physiologische Protoplasmastoffwechsel und die Purinbildung. Arch. f. d. ges. Physiol. Bd. 134 S. 59-102. 1910.

3) F. Mare š, Sur l'origine de l'acide urique chez l'homme. Arch. slaves de biol. t. 3 p. 222.1887.

Pflüger's Archiv für Physiologie. Bd. 155. 
des échanges moléculaires dans le protoplasma, dans l'albumine organisée, tandis que l'urée provient de la destruction des matières azotées, ingérées dans les aliments et absorbées, et de l'albumine en circulation. Les échanges moléculaires dans le protoplasma en repos, surtout celui des cellules glandulaires, produisent une certaine quantité d'acide urique, dépendant de l'individualité et de l'âge du protoplasma. La quantité d'urée formée dans le même temps, dépend de la quantité des matières azotées, ingérées et absorbées.

Eine Lösung der speziellen chemischen Frage, welche Sub . stanz sich in der Zelle bei der Bildung der Harnsäure beteiligt, konnte zu dem Plan der physiologischen Untersuchungen von Mareš im Jahre 1886 und 1887 nicht angehören. Dies konnte erst die spätere Forschung entscheiden. Da kam die Zeit der Arbeiten von Kossel, die den Zusammenhang zwischen Xanthinbasen und Nukleinen entdeckten, und der Untersuchungen von Horbaczewski ${ }^{1}$, , dem das hohe Verdienst angehört, im Jahre 1889 und 1891 die genetische Beziehung der Harnsäure zu den Xanthinbasen und Nukleinen nachgewiesen zu haben, so dass das Nuklein der Zellen als die Muttersubstanz der Harnsäure anzusehen wäre. Infolgedessen formulierte $\mathrm{Mareš}^{2}$ ) seine Theorie der Bildung der Harnsäure im Säugetierorganismus wie folgt:

Die Harnsäure ist ein Produkt des Stoffwechsels in den lebenden Körperzellen, wobei namentlich die Nukleine der.Zellenkerne beteiligt sind.

Diese allgemeinen Sätze bilden das Resultat der ursprünglichen 22 Versuche, welche der genannte Autor im Jahre 1886 und 1887 an sechs in nüchternen Zustand versetzten Männern im Alter von 13-45 Jahren angestellt hat, deren Beschreibung hier kurz in seiner eigenen, in seinen beiden diesbezüglichen Arbeiten vorkommenden Schilderung wiedergegeben sei.

Die Versuche begannen 12 Stunden nach der letzten Nahrungsaufnahme und dauerten bis zur 24., einige Male bis zur 27. Stunde. Der Harn wurde in dreistündigen Perioden gesammelt.

Die Harnsäure wurde nach Salk ow ski - Lu d wig, der Gesamtstickstoff nach $\mathrm{Kjeldahl} \mathrm{bestimmt.}$

1) J. Horbaczewski, Sitzungsber. d. kais. Akad. in Wien Bd. 98, Juli 1889 ; Bd. 100, April 1891.

2) F. Marě̌, Zur Theorie der Harnsäurebildung im Säugetierorganismus. Sitzungsber. d. kais. Akad. in Wien Bd. 101 S. 10, Januar 1892. 
Ein experim. Beitrag zur Lehre von der individuellen Konstanz etc. 525

Diese Versuche ergaben, dass im nüchternen Zustande die von einer Versuchsperson ausgeschiedene Harnstickstoffmenge an verschiedenen Tagen in sehr weiten Grenzen bis nahezu um $100 \%$ variieren kann, dass dagegen die zur gleichen Zeit ausgeschiedene Harnsäuremenge nur wenig um einen Mittelwert herumschwankt, welcher bei verschiedenen Versuchspersonen verschieden ist und individuel] so konstant erscheint, dass ihn Mareš zuerst als eine Harnsäurekonstante, "constante d'acide urique" ${ }^{1}$ ), und sozusagen spezifisch für die betreffende Person "pour ainsi dire spézifique pour chaque individue $\left.{ }^{1}\right)^{2}$ ) bezeichnete. So variert bei der Versuchsperson A die Harnstickstoffausscheidung in 15 Stunden nüchternen Zustandes an verschiedenen Tagen zwischen 6078 bis $10005 \mathrm{mg}$, die Harnsäuremenge aber erscheint zu gleicher Zeit, a n ziemlich weit entfernten Tagen, ganz gleichmässig. Dass diese zeitliche Entfernung eine beträchtliche war, sei hier besonders hervorgehoben. Die erwähnte Versuchsperson A schied am 16. Juni 1886 (Versuch I) in 15 Stunden nüchternen Zustandes $287 \mathrm{mg}$ Harnsäure aus, am 14. Dezember 1886 (Versuch XI), also ein halbes Jahr später, schied sie unter gleichen Versuchsbedingungen $273,6 \mathrm{mg}$ aus. Also eine nahezu gleiche Menge von Harnsäure ${ }^{3}$ ). In allen elf Versuchen, die an der Person A im Verlaufe des erwähnten halben Jahres unter gleichen Bedingungen vorgenommen wurden, hält sich die Menge der ausgeschiedenen Harnsäure sehr nahe am Durchschnittswerte von $\mathbf{2 6 5 9 9} \mathrm{mg}$, woraus sich für diese Versuchsperson ein individuell konstanter Wert von 17,7 mg Harnsäure in einer Stunde ergibt. An der Versuchsperson B wurden drei Versuche (XII-XIV) im Verlaufe eines Monates ausgeführt. Die Harnstickstoffausscheidung schwankt im nüchternen Zustande zwischen 5148-9471 mg für 15 Stunden. Die Harnsäuremenge beträgt am 27. November 1886 (Versuch XII) für 15 Stunden nüchternen Zustandes 334,2 $\mathrm{mg}$, am 20. Dezember 1886 (Versucb XIV), also beinahe um einen Monat später, unter denselben Versuchs-

1) F. Mare s, Sur l'origine de l'acide urique chez l'homme. Arch. slaves de biol. t. 3 p. 212, 213, 215, 221. 1887.

2) F. Marěs, Zentralbl. f. d. med. Wissensch. Jałrg. 26, 1886 S. 2. Ref. J. Munk. - E. Schreiber, Über die Harnsäure unter physiologischen und pathologischen Bedingungen S. 32. Stuttgart 1899.

3) F. Mareš, Der physiologische Protoplasmastoff'wechsel und die Purinbildung. Arch. f. d. ges. Physiol. Bd. 134 S. 64-66. 1910. 
bedingungen $369 \mathrm{mg}$, der Durchschnittswert derselben hält sich knapp um 345,8 $\mathrm{mg}$ oder $\mathbf{2 3} \mathrm{mg}$ in einer Stunde. Die weiteren Versuche (XVI-XXII), welche an weiteren vier Männern (C.-F.) in kleineren Zeitabständen ausgeführt wurden, ergahen: für Versuchsperson C. Harnstickstoff $5674-6896 \mathrm{mg}$, Harnsäure $\mathbf{3 0 5 , 0} \mathrm{mg}$ für 12 Stunden, d. h. 25,4 mg pro Stunde; für Versuchsperson D Harnstickstoff $3472-6849 \mathrm{mg}$, Harnsäure 182,7 mg für 12 Stunden, $\mathbf{1 5 , 2} \mathrm{mg}$ pro Stunde; für Versuchsperson E. Harnstickstoff 2891 bis $5548 \mathrm{mg}$, Harnsäure 161,9 $\mathrm{mg}$ in 12 Stunden, 13,5 $\mathrm{mg}$ in 1 Stunde; für Versuchsperson F. Harnstickstoff 4213-6097 mg, Harnsäure $183,9 \mathrm{mg}$ in 12 Stunden, $15,3 \mathrm{mg}$ in 1 Stunde.

„Die grossen Variationen in der Harnstickstoffausscheidung wurden absichtlich dadurch hervorgerufen, dass die Versucbsperson mit der letzten Mahlzeit, 12 Stunden vor Beginn eines jeden Versuches, das einemal sehr wenig, das anderemal sehr viel Eiweiss (Fleisch) zu sich genommen hatte. Der Einfluss dieser eiweissreichen Nahrung zeigte sich von der 12,-24. Stunde nach deren Einnahme noch sehr deutlich in der Harnstickstoffmenge (Harnstoff); auf die Harnsäuremenge aber war zu dieser Zeitperiode kein Einfluss mehr zu beobachten. Danach erscheint die Harnsäuremenge von der eingenommenen Eiweiss(Fleisch-) menge ganz unabhängig zu einer Zeitperiode, wo der Einfluss dieser Eiweissmenge auf die Menge des Harnstickstoffes (Harustoffe's) noch sehr deutlich ist" ${ }^{\text {}}$ ). Dies ist ein schwerwiegendes Zeugnis für eine selbständige Herkunft der Harnsäure, die offenbar aus einer anderen, individuell verfärbten Quelle entspringt.

Diese Tatsachen wurden später, wie Mar eš sagt ${ }^{1}$ ), von anderen Untersuchern bestätigt, wiewohl (auch) unter anderen Versuchsbedingungen. So weist Salkowski²) (nach Spilker's im Stickstoffyleichgewicht ausgeführten Versuchen) darauf hin, dass ein Parallelismus zwischen der ausgeschiedenen Harnsäure und Harnstoff keineswegs besteht, und setzt fort:

1) F. Mare ̌̌, Der physiologische Protoplasmastoffwechsel und die Purinbildung. Arch. f. d. ges. Physiol. Bd. 134 S. 66. 1910.

2) E. Salkowski, Über die Grösse der Harnsäureausscheidung und den Einfluss der Alkalien anf dieselbe. (Nach Versuchen von Dr. E. Spilker.) Virchow's Arch. f. path. Anat. u. Physiol. u. f. klin. Med. Bd. 117 ,S. 570 bis 581. 1889. 
„Diese Erfahrungen sprechen sehr zugunsten der Ausicht von Mare $\check{s}$, dass die Quantität der Harnsäure ihrem grösseren Teile nach einen persönlichen individuellen Wert darstellt, der von der Nahrung nur wenig beeinflusst wird. Nach seiner Ansicht bildet sie sich nur aus dem organischen Eiweiss, nicht aus dem Nahrungseiweiss, wenn dieses den Stoffwechselvorgängen verfällt, ohne vorher organisiert zu sein." Im weiteren zeigt er auf die bedeutenden Schwankungen des Verhältnisses Harnsäure : Harnstoff in den älteren Angaben, in welchen doch gleichfalls ein Argument dafür liegt, „dass die Quantität der ausgeschiedenen Harnsäure von im Individuum selbst, nicht in der Nahrung liegenden Verhältnissen abhängt."

Einen sehr untergeordneten Einfluss der Eiweisszufuhr auf die Grösse der (täglichen) Harnsäureausfuhr deduziert Salkowski daselbst ${ }^{1}$ ) aus den Mittelwerten, welche er hier aus Hirschfeld's ${ }^{2}$ ) zu anderen Zwecken angestellten Versuchen berechnet. Hirschfeld's Versuche wurden in fünf Reihen ausgeführt, und die Mittelwerte der Harnsäure pro Tag zeigen nur geringfügige Schwankungen. Weiter rät dann Salkowski, sich für klinische Zwecke um die Relation zwischen Harnsäure unḍ Harnstoff überhaupt nicht mehr zu kümmern und statt dessen auf die absolute Grösse der täglichen Harnsäureexkretion zurückzugehen. Im Autoreferate ${ }^{3}$ ) über diese seine und Spilker's Arbeit bezeichnet Salkowski die Harnstoffausscheidung als in höchstem Grade abhängig von der Grösse der Eiweisszufuhr; von der Harnsäureausscheidung drückt er sich folgendermaassen aus: "Die absolute Grösse derselben ist ohne Zweifel individuell, wie aus jetzt schon ziemlich zahlreichen, nach guten Methoden ausgeführten Bestimmungen hervorgeht." Ähnlich urteilt auch Noorden ${ }^{4}$, welcher in seinem Lehrbuch bei den Erwägungen über die Schwankungen des Quotienten Gesamt-N und somit seine Unverlässlichkeit als $\frac{\text { Gesamt-N }}{\text { Harnsäure-N }}$ Maassstab über die

1) E. Salkowski, l. c. S. 574 .

2) F. Hirschfeld, Beiträge zur Ernährungslehre des Menschen. Virchow's Arch. f. path. Anat. u. Physiol. u. f. klin. Ned. Bd. 114 S. 301-340. 1888.

3) E. Salkowski, Über die Grösse der Harnsäureausscheidung und den Einfluss der Alkalien auf dieselbe. Autoreferat im Zentralbl. f. d. med. Wissenschaften Jahrg. 28 S. $360-361$.

4) C. v. Noorden, Lehrbuch der Pathologie des Stoffwechsels S. 54-55. Berlin 1893. 
Harnsäureausscheidung zu dienen sich äussert: „Einen besseren Maassstab gewährt die absolute Menge der Harnsäure. Es wird jetzt von verschiedenen Seiten (Marě̌s, Salkowski), wie mir nach eigenen Erfahrungen scheint - mit Recht gelehrt (von mir unterstrichen), dass für die Menge der Harnsäure individuelle Verbältnisse an erster Stelle maassgebend sind. Der eine menschliche Organismus liefert stets hohe, der andere stets kleine Harnsäurewerte; die Zähigkeit, mit welcher an denselben festgehalten wird (Hirschfeld!) imponiert viel mehr als die kleinen Änderungen, welche man durch Wechsel der Nahrung, des Getränkes usw. erzwingen kann." Auch in der Abhandlung daselbst über die physiologische Beeinflussung der Harnsäureausscheidung durch die Nahrung erwähnt er, dass „neue Gesichtspunkte für die Beziehungen zwischen Nahrung und Harnsäure sich ergeben aus den Untersuchungen von Mareš und Horbaczewski" und sagt: "Nach Mares stellt sich die Harnsäureausscheidung ca. 13 Stunden nach der letzten Mablzeit, also im nüchternen Zustande auf einen zwar individuell verschiedenen, aber für jedes Individuum von Stunde zu Stunde (es ward bis zur 27. Stunde untersucht) zunächst konstant bleibenden Minimalwert ein." "Aus diesem Minimalwert erhebt sich die Ausscheidung 2-5 Stunden nach einer Mahlzeit - um das Doppelte und Dreifache pro Stunde nach Fleischgenuss, (um ein geringeres nach vegetabilischer Kost.") (Von mir eingeklammert, da in den Versuchen von Mareš der Einfluss der vegetabilischen Kost nicht geprüft wurde.)

Die von M a rěs herrührende Erkenntnis der individuellen Konstanz ist somit zu einer Lehre geworden, wie dies auch weiter aus Schreiber's') Monographie über die Harnsäure einleuchtet. Nach Schreiber hat zwar "die Theorie Mareš, dass die Ür aus molekularen Veränderungen des Zellprotoplasmas, insbesondere der Verdauungsdrüsen, entstehe, wohl nie ernste Anerkennung gefunden"; aber derselbe führt an, „dass die Grösse der Harnsäureausscheidung individuell sehr verschieden, für das einzelne Individuum aber konstant, fast spezifisch ist', haben uns die Untersuchungen M a re š's gelehrt," und meint, "man könnte diese (d. i. die individuell konstante Menge der Harnsäure ausdrückende) Zahl weit eber als ,individuelle Mittelzahl" bezeichnen". Des weiteren waren es insbesondere

1) E. Schreiber, Über die Harnsäure unter physiologischen und pathologischen Bedingungen S. 31, 32, 81. Stuttgart 1899. 
Burian und Schur ${ }^{2}$, die auf Grund eigener Versuche die Lehre von der individuellen Koustanz der Harnsäureausfuhr bekräftigten. Ihre diesbezüglichen Versuche wurden zwar im Stickstoffgleichgewicht bei fixer Lebensweise unter abermạliger Aufnahme einer purinfreien Kost während des Tages ausgeführt, jedoch sie wurden ähnlich wie die Versuche Mareš's an demselben Individuum in weit a useinanderliegenden Zeitpunkten, im Mai (August) resp. November 1899 angestellt. Die eine Reihe der Versuche (vom Mai) lieferte als Mittelwert des täglich eliminierten Harnpurin - N $\mathbf{0 , 1 9 9} \mathrm{g}$, die andere (vom November), nach $\mathbf{5}$ Monaten, brachte 0,200 g als Mittelwert für die 24 stündliche endogene Harnpurin-N-Menge, welche Zahl auch mit der Mittelzahl der Augustexperimente, die unter vegetabilischer Diät geschahen, übereinstimmte. Dadurch veranlasst, gingen die genannten Autoren so weit, dass sie sich nicht nur für die individuelle Konstanz des endogenen Harnpurinwertes aussprachen, sondern denselben sogar für ein und dasselbe Individuum als eine physiologische Konstantebezeichneten.

Weitere Belege für die individuelle Konstanz der Harnsäureausfuhr unter sonst gleichen Verhältnissen, auch bei mehrmaliger $\mathrm{Nahrungsaufnahme}$ während des Tages, findet man in den Arbeiten einer ganzen Reihe anderer Forscher: Minkowski²), Schreiber und Waldvogel ${ }^{3}$ ), Sivén ${ }^{4}$ ), besonders Rockwood ${ }^{5}$ ), der an einem Individuum 6 Serien von Bestimmungen zu verschiedenen Zeitperioden eines ganzen Jahres, an einem anderen 2 annähernd zwei Monate von einander entfernte Serien ausführte, u. a.

Die individuelle Konstanz der Harnsäure im nüchternen Zustande mit einmaliger purinfreien Nahrungsaufnahme während 24 Stunden bezeugen zwei Versuche Smetánka's ${ }^{6}$, die zu anderen Zwecken an einem und demselben Individuum im Zeitabstand von 7 Wochen ausgeführt wurden, und wo im ersten $378,7 \mathrm{mg}$,

1) R. Burian und H. Scbur, Über die Stellung der Purinkörper im menschlichen Stoffwechsel. Arch. f. d. ges. Physiol. Bd. 80 S. 241. 1900.

2) O. Mink ow ski, Arch. f. exper. Path. u. Pharm. Bd. 41 S. 403. 1898.

3) Schreiber und Waldvogel, Arch. f. exper. Path. u. Pharm. Bd. 42. 1899 .

4) V. O. Sivé n, Skandin. Arch. f. Physiol. Bd. 11 S. 123.1901.

5) E. Rockwood, Americ. Journ. of Physiol. vol. 12 p. 38.1904.

6) F. Smetánka, Zur Herkunft der Harnsäure beim Menschen. Arch. f. d. ges. Physiol. Bd. 138 S. 226, 229, 267. 1911. 
im zweiten 370,2 $\mathrm{mg}$ Harnsäure, also nur etwa um 2\% weniger, ausgeschieden wurden, welcher Umstand demselben Autor nicht entging. Wir werden dieser Versuchsanordnung noch bei eigenen Versuchen im Sinue der Lehre von der Harnsäurekonstanz begegnen.

Die am Anfange dieser Arbeit angeführten allgemeinen Sätze von Mareš über die Herkunft der Harnsäure bei Menschen enthalten die Beantwortung der Frage, die sich der genannte Autor legte ${ }^{1}$ ), nachdem er deren Konstanz feststellte: mit welchen Umständen könnte die bei verschiedenen Personen verschiedene individuelle Grösse des Harnsäurewertes zusammenhängen? Wenn sie mit der Aktivität des Protoplasma im Zusammenhang wäre, so könnte das Alter der Versuchspersonen und die dureh dasselbe bedingte Änderung der Aktivität von Einfluss sein. Dieser seinen Erwägung entsprechend stellte Mareš seine Versuchsergebnisse in eine Tabelle zusammen, wobei er den individuellen Harnsäurewert jeder Versuchsperson für $1 \mathrm{~kg}$ Körpergewicht und 24 Stunden berechnete und diese Werte nach dem Alter der Versuchspersonen ordnete ${ }^{2}$ ).

Diese Tabelle gestaltete sich folgendermaassen:

\begin{tabular}{|c|c|c|c|}
\hline $\begin{array}{l}\text { Versuchs- } \\
\text { person }\end{array}$ & $\begin{array}{l}\text { Alter } \\
\text { Jahre }\end{array}$ & $\begin{array}{c}\text { Körper- } \\
\text { gewicht } \\
\text { kg }\end{array}$ & $\begin{array}{l}\text { Harnsäure- } \\
\text { wert für l kg } \\
24 \text { Stunden } \\
\text { mg }\end{array}$ \\
\hline $\begin{array}{l}\mathrm{E} \\
\mathrm{F} \\
\mathrm{D} \\
\mathrm{B} \\
\mathrm{C} \\
\mathrm{A}\end{array}$ & $\begin{array}{l}13 \\
16 \\
16 \\
26 \\
30 \\
45\end{array}$ & $\begin{array}{l}38 \\
41 \\
58 \\
56 \\
70 \\
57\end{array}$ & $\begin{array}{l}8,53 \\
8,96 \\
6,29 \\
9,86 \\
8,78 \\
7,40\end{array}$ \\
\hline
\end{tabular}

Wenn man vorläufig von der Versuchsperson D absieht, so kanu man aus der Tabelle ein Steigen des individuellen Harnsäurewertes bis zum 26. Lebensjahre, also bis zur Vollendung des Wachstums, herauslesen und dann mit steigendem Lebensalter ein Herabsinken desselben und dieses Verhältnis mit der Körperentwicklung in Zusammenhang bringen. Aber „zur Behauptung

1) F. Marě, Sur l'origine de l'acide urique chez l'homme. Arch. slaves de biol. t. 3 p. 215, 216, 218, 226. 1887.

2) F. Mareš, Der physiologische Protoplasmastoffwechsel und die Purinbildung. Arch. f. d. ges. Physiol. Bd. 134 S. 68. 1910. 
Ein experim. Beitrag zur Lehre von der individuellen Konstanz etc. 531

eines solchen Verhältnisses genügen diese Versuche nicht; es mü ss ten solcheVersuche an einer und derselben Versuchsperson im verschiedenen Alter angestellt werden, was nicht leicht $\mathrm{zu}$ verwirklichen ist" ${ }^{1}$ ), sagt M are š (die Unterstriche rühren von mir her).

Bei der Person D, welche eine zum Körpergewicht übermässige Körperlänge $(58 \mathrm{~kg}-1,72 \mathrm{~m})$ and somit ein Übergewicht des Skelettes über die parenchymatösen Organe aufweist, ist jenes Verhältnis durch ungleichmässige Entwicklung im Vergleiche zu der auch im 16. Lebensjahre stehenden Person F verdeckt, so dass ihr niedriger Harnsäurewert sich eben wieder aus dem Zusammenhange mit der Tätigkeit der protoplasmatischen Organe begreifen liesse.

Die Aktivität des Protoplasmas ist wohl bei der Bildung und dem Wachstum des Körpers am intensivsten. Im Einklange damit fand Mareš wohl als erster die Harnsäureproduktion beim Neugeborenen tatsächlich ungemein gross ${ }^{2}$ ). Seine diesbezüglichen Untersuchungen an den Neugeborenen von den ersten Lebensstunden bis etwa zum 14. Lebenstage ergaben, dass in den ersten Lebensstunden mehr als $8 \%$ des gesamten Harnstickstoffes in Form von Harnsäure ausgeschieden werden, während dieses Verhältnis beim Erwachsenen kaum $2 \%$ erreichen dürfte. In den folgenden Tagen sinkt dieses Verhältnis, die Harnsäureproduktion vermindert sich.

Diese Befunde an den Neugeborenen, wo das Wachstum am raschesten ist, wo eine rasche karyokinetische Zellyermehrung zweifelsohne stattfindet, wo die Aktivität des Protoplasmas im Vergleiche $\mathrm{zu}$ derjenigen eines erwachsenen Organismus als eine gesteigerte, erhöhte aufzufassen ist, ergeben im allgemeinen die Tatsache: mit der Steigerung der physiologischen Tätigkeit der Körperzellen, soferne dieselbe mit molekularen, stofflichen Änderungen im Protoplasma zusammenhängt, steigt die Harnsäurebildung ${ }^{3}$ ).

Speziell ist diese Tatsache dort zu bemerken, wo man die Zellen bestimmter Organe experimentell zu einer erhöhten Tätigkeit

1) F. Mares, Iler physiologische Protoplasmastoffwechsel and die Purinbildung. Arch. f. d. ges. Physiol. Bd. 134 S. 69.1910.

2) F. Mareš, Sur l'origine de l'acide urique chez l'homme. Arch. slav. de biol. t. 3 p. 217. 1887. - Der physiologische Protoplasmastoffwechsel und die Puriubildung. Arch. f. d. ges. Physiol. Bd. 134 S. 69.1910.

3) F. Mare ̌, Arch. f. d. ges. Physiol. Bd. 134 S. 98 u. 99.1910. 
anregen kann, welche sich eben durch sichtbare stofflichen Änderungen im Protplasma kundgibt. Dies ist bei den Verdauungsdrüsen der Fall, welche man auf natürliche Weise durch Nahrungsaufnahme zur Tätigkeit anregen kann, wonach eine Steigerung der Harnsäureausfuhr eintritt. Somit sind wir zu der zweiten tatsächlichen Grundlage der Theorie von Mareš gekommen: zur Harnsäurevermehrung nach dem Fleischgenuss, welche ihrem zeitlichen Verlaufe nach mit der Tätigkeit der Verdauungsdrüsen einhergeht, wogegen sich die damit verbundene Harnstickstoffvermehrung später einstellt und länger währt. Die Resultate der späteren Forscher, die nach Darreichung einer purinfreien Nabrung keine Harnsäurevermehrung zu finden glaubten und so den Einwand erhoben, dass dieselbe von den Nahrungspurinen herrührt, sind durch die Arbeit S m etán ka's ${ }^{1}$ ) entkräftet worden, welcher mit einwandfreier Methode den Nachweis lieferte, dass tatsächlich auch purinfreie Nahrungsstoffe, besonders die Proteine, eine deutliche Harnsäuremehrung hervorrufen, worlurch der $\mathrm{z}$ we.ite tatsächliche Grund der Theorie von Mare š befestigt und erweitert wurde.

Endlich kommen wir zu dem dritten faktischen Grund dieser Theorie: zu der Harnsäurevermehrung nach der in acht Versuchen bei vier Personen künstlich durch das Pilokarpin hervorgerufenen Drüsentätigkeit, wobei die zeitlichen Verhältnisse entsprechen, und wobei auch der spätere Nachweis eines den intensiven Nukleinmetabolismus bezeugenden nukleinbaltigen Sekretes aus dem Pankreas bei dieser Tätigkeit schwer zugunsten der Theorie ins Gewicht fällt. So konnte M areš wirklich die ausgeschiedene Harnsäuremenge für ein Maass des physiologischen Stoffwechsels, "la mesure des échanges moléculaires du protoplasma" ${ }^{2}$ ), halten.

Wenden wir nun wieder die Aufmerksamkeit der Tatsache der individuellen Konstanz der Harnsäuremenge beim Menschen zu, welche von Mareš zuerst im nüchternen Zustande nachgewiesen wurde, und welche die erste faktische Grundlage seiner Theorie der Entstehung von Harnsäure beim Menschen bildet. Wie wir gesehen haben, ist der Erkenntnis von der Konstanz der Harnsäure eine fast

1) F. Smetánka, Zur Herkunft der Harnsäure beim Menschen. Arch. f. d. ges. Physiol. Bd. 138 S. 217-274. 1911.

2) F. Marěs, Sur l'origine de l'acide urique chez l'homme. Arch. slaves de biol. t. 3 p. 226.1887. 
Ein experim. Beitrag zur Lehre von der individuellen Konstanz etc. 533

allgemeine Anerkennung zuteil geworden - Ausnahme macht vielleicht nur Fol in ${ }^{1}$ ) - , so dass sie als eine Leh re betrachtet werden kann. Obzwar sie als eine solche keine überzähligen Beweise brancht, würden doch immer Experimente von Interesse und Wichtigkeit sein, die an einem und demselben Individuum unter vollständig gleichen Versuchsbedingungen in möglichst grossen Zeitabständen ausgeführt wären. Am idealsten wäre es, solche Experimente an derselben Versuchsperson in verschiedenem Alter, von der Jugend an bis in das Greisenalter, anzustellen. So könnte man vielleicht dann behaupten, dass das aus der oben angeführten Tabelle, die eine Vergleichung der individuellen Harnsäurewerte nach dem Alter und dem Körpergewicht der Versuchspersonen darstellt, erschlossene Steigen des individuellen Harnsäurewertes bis zum 26. Lebensjahre, das Herabsinken desselben mit dem Fortschreiten des Lebensalters - und diesen Umstand durch die Körperentwicklung - durch die im Alter sich ändernde Aktivität des Protoplasmas zu erklären ist. $\mathrm{Zu}$ solcben Frwägungen veranlassen auch die Resultate $\mathrm{T}$ an $0^{\prime} \mathrm{s}^{2}$ ), dessen Arbeit mir als Dissertation im Original nicht zugänglich war. Aber nach der Beschreibung bei Schreiber fand er gelegentlich der Untersuchung über den Leukocytengehalt des Blutes und die Harnsäuremenge im Harn in den verschiedenen Lebensaltern, dass die Ur-menge in der ersten Lebenszeit relativ hoch ist, dann fällt, um gegen das 20. Jahr wieder zu steigen; zwischen dem 20. und 30. Lebensjahre erreicht sie die grösste Höhe und nimmt dann wieder ab. ("Es werden noch weitere Untersuchungen in dieser Richtung nötig sein", bemerkt Schreiber.) Solche Experimente sind, wie schon erwähnt, vorläufig nicht leicht zu verwirklichen.

Eine Konstanz ist um so fester, in je grösserer zeitlichen Distanz ihre Feststellung an einem und demselben Subjekte unter sonst gleichen Verhältnissen stattfindet. Deswegen habe ich schon am Anfang den beträchtlichen Zeitabstand der ursprünglichen Versuche M areš' hervorgehoben. Aus diesen allgemeinen Gründen versuchte ich es, einen von den Männern, die bei den ursprünglichen Untersuchungen Mareš' im Jahre 1886 als Versuchspersonen dienten,

1) Fol in, The Americ. Journ. of Physiol. vol. 13 p. 66.1905.

2) Schreiber, Über die Harnsäure unter physiologischen und pathologischen Bedingungen S. 30. Stuttgart 1899. 
zu einer neuen Versuchsreihe zu bewegen. Dies gelang im Jahre 1911, also nach $25 \mathrm{Jahren}$.

Speziell verfolgte ich dann noch einen Gedankengang angesichts der neuen Versuche an diesem aus der damaligen Reihe noch zugänglichen Subjekte. Es war der Mann A, an dem viele, zusammen elf Versuche, angestellt wurden. Damals im Jabre 1886 stand er im Mannesalter von 45 Jahren. Nun im Jahre 1911 ist er ein 70jähriger Greis. Was die qualitativen Verbältnisse des Stoffwechsels im Greisenalter anbelangt, sind keine Abweichungen von dem des Mannesalters bekannt; über die quantitativen Verhältnisse gibt es aber ziemlich spärliche Angaben. Ausser den drei Fällen Limbeck's ${ }^{1}$ ), deren Resultate sich widersprechen, da in einem die Menge der Harnsäure zu hoch, in Mittel 1,23 g, in den zwei anderen niedrige oder normale Mengen $0,14-0,28 \mathrm{~g}$ waren, ist so viel wie gar nichts bekannt, so dass man sagen kann, dass das Verhalten der Harnsäureausscheidung im Greisenalter noch zu untersuchen ist. Und zu dieser Frage wollte ich experimentell auch beitragen. Ich fragte mich dabei, wenn ich die Ausscheidung der Harnsäure, im Lichte der Claude-Bernard'schen Auffassung der Lebensvorgänge, als einen dissimilatorischen Vorgang betrachten soll, ob ich nicht im Greisenalter, welches sich durch eine vermehrte Dissimilation im Verhältnis zur Assimilation auszeichnet, eher eine Vermehrung statt eine Verminderung der Harnsäureproduktion erwarten könnte? Und ob die neue Versuchsreihe in dieser Auffassung einen Beitrag zur Physiologie des Alters nicht liefern könnte?

Was die Anordnung der neuen Versuchsreihe anbelangt, sei hier auf folgendes aufmerksam gemacht.

Diesmal wurden nur Tagesversuche von $z$ wölstüdlicher Dauer angestellt. Dies geschah erstens deswegen, damit die nächtlichen Schwankungen der Harnsäureausscheidung ausgeschlossen werden. Denn, wie bekannt, sind die Mengen der Harnsäure, die in dreistündigen Phasen einer zwölf- bis fünfzehnstündlicher Periode des $\mathrm{n} u \mathrm{ch}$ ternen $\mathrm{Zustandes}$ ausgeschieden werden und zusammen für jede Versuchsperson einen konstanten Wert ausmachen, nicht gleichmässig; die abendlichen und nächtliehen Harnsäuremengen sind geringer als die während des Tages ausgeschiedenen,

1) C. v. Noorden, Handbuch der Pathologie des Stoffwechsels, 2. Aufl, Bd. 1. 1906. Daselbst Magnus-Levy, Physiologie des Stoffwechsels. G. Der Stoffwechsel im Greisenalter S. 472. 
Ein experim. Beitrag zur Lehre von der individuellen Konstanz etc. 535

wobei der Höhepunkt in die Vormittagsstunden fällt, so dass man von einer physiologischen Retention der Harnsäure sprechen kann. Die Verhältnisse, auf die Sivén ${ }^{1}$ ) zum ersten Male aufmerksam machte, wurden von allen Autoren, die sich mit der Harnsäureausscheidung phasenweise beschäftigten, bestätigt. So von $\mathrm{Pfeil}^{2}$ ), Rockwood ${ }^{3}$, Leathes ${ }^{4}$ ), Hirschstein ${ }^{5}$ ), Kennaway ${ }^{6}$ ), Smetánka ${ }^{7}$ ). Zweitens geschab das aus Rücksicht auf die Bequemlichkeit der nunmehr bejahrten Versuchsperson. Deswegen wurde auch die Versuchsdauer auf 12 Stunden beschränkt. Die Versuchsperson wurde in nüchternen Zustand versetzt, da die letzte Nahrungsaufnahme 12 Stunden vor Anfang des Versuches, immer um 6 Uhr abends an dem Tage zuvor, stattfand. Der eigentliche Versuch fing um. 6 Uhr früh an und endete um 6 Uhr abends. Da mir die Protokolle vom Jahre 1886 zur Verfügung standen, konnte ich bei der Versuchsperson alles genau so einrichten, wie es vor 25 Jahren war. Aus den angeführten Gründen müssen sun aus der Reihe von XI 15 stündigen Versuchen aus dem Jabre 1886, die da als Kontrollversuchsreihe angeführt sind, die zwei nächtlichen Versuche Nr. III und IV ausgeschlossen werden und die zwölfstündigen Gesamtmengen aus den korrespondierenden Phasen berechnet werden. Es folgen nun die Originaltabellen der neun Tagesversuche aus dem Jahre $1886^{8}$ ), die nun nicht nur die dreistündigen Phasen und die 15 stündigen Gesamtmengen, sondern auch die zwölfstündigen Gesamtmengen der Harnsäure enthalten. Eine Übersichtstabelle von diesen zwölfstündigen Werten samt dem Durchschnittswerte für 1 Tag und 1 Stunde ist beigefügt.

\section{Versuche vom Jahre 1886.}

Versuchsperson A. Mann von 45 Jahren. Körpergewicht $57 \mathrm{~kg}$. Körperlänge 1,57 m. Anfang der Versuche immer um $6 \mathrm{Uhr}$ früh.

1) V. O. Sivén, Skand. Arch. f. Physiol. Bd. 11 S. 123.1901.

2) P. Pfeil, Zeitschr. f. physiol. Chemie Bd. 40. S. 1. 1903/4.

3) E. Rocw o od, Americ. Journ. of Physiol. vol. 12. p. 46, 47. 1904.

4) I. B. Leathes, Journ. of Physiol. vol. 35. p. 125. 1907.

5) L. Hirschstein, Arch. f. experim. Pathol. u. Pharmakol. Bd. 57. S. 229.1907.

6) E. L. Kennaway, Journ. of Physiol. vol. 38. p. 1. 1908.

7) F. Smetánka, Arch. f. d. ges. Physiol. Bd. 138 S. 217. 1911.

8) F. Mareš, Sur l'origine de l'acide urique chez l'homme. Arch. slaves de biol. t. 3 p. 208. Paris 1887. 
I. 16. Juni 1886 .

\begin{tabular}{|c|c|c|c|c|c|c|c|}
\hline Zahl & $\begin{array}{c}\text { Tages- } \\
\text { zeit } \\
\text { Uhr }\end{array}$ & $\begin{array}{c}\text { Harn- } \\
\text { menge } \\
\text { ccm }\end{array}$ & $\begin{array}{c}\text { Harn- } \\
\text { stickstoff } \\
\text { mg }\end{array}$ & $\begin{array}{c}\text { Gesamt- } \\
\text { menge in } \\
12 \text { Stdn. } \\
\text { mg }\end{array}$ & $\begin{array}{c}\text { Harn- } \\
\text { säure } \\
\text { mg }\end{array}$ & $\begin{array}{c}\text { Gesamt- } \\
\text { menge in } \\
12 \text { stdn. } \\
\mathrm{mg}\end{array}$ & $\begin{array}{c}\text { Anteil der } \\
\text { Harnsäure } \\
\text { a. Gesamt- } \\
\text { stickstoff } \\
\%\end{array}$ \\
\hline $\begin{array}{l}1 . \\
2 . \\
3 . \\
4 . \\
5 .\end{array}$ & $\begin{array}{c}6-9 \\
9-12 \\
12-3 \\
3-6 \\
6-9\end{array}$ & $\begin{array}{l}490 \\
260 \\
240 \\
440 \\
170\end{array}$ & $\begin{array}{l}1894 \\
1492 \\
1321 \\
1397 \\
1010\end{array}$ & 6104 & $\begin{array}{l}94,0 \\
83,7 \\
35,7 \\
34,5 \\
39,1\end{array}$ & 247,9 & $\begin{array}{l}1,65 \\
1,87 \\
0,90 \\
0,82 \\
1,29\end{array}$ \\
\hline 1 & & 400 & 7114 & - & 287,0 & - & 1,34 \\
\hline
\end{tabular}

II. 22. Juni 1886 .

\begin{tabular}{|c|c|c|c|c|c|c|c|}
\hline Zahl & $\begin{array}{c}\text { Tages- } \\
\text { zeit } \\
\text { Chr }\end{array}$ & $\begin{array}{l}\text { Harn- } \\
\text { menge } \\
\text { ccm }\end{array}$ & $\begin{array}{c}\text { Harn- } \\
\text { stickstoff } \\
\text { mg }\end{array}$ & $\begin{array}{c}\text { Gesamt- } \\
\text { menge in } \\
12 \text { Stdn. } \\
\mathrm{mg}\end{array}$ & $\begin{array}{c}\text { Harn- } \\
\text { säure } \\
\text { mg }\end{array}$ & $\begin{array}{c}\text { Gesamt- } \\
\text { menge in } \\
12 \text { Stdn. } \\
\text { mg }\end{array}$ & $\begin{array}{l}\text { Anteil der } \\
\text { Harnsäure } \\
\text { a. Gesamt- } \\
\text { stickstoff } \\
\%\end{array}$ \\
\hline $\begin{array}{l}1 . \\
2 . \\
3 . \\
4 . \\
5 .\end{array}$ & $\begin{array}{c}6-9 \\
9-12 \\
12-3 \\
3-6 \\
6-9\end{array}$ & $\begin{array}{l}230 \\
180 \\
230 \\
200 \\
130\end{array}$ & $\begin{array}{l}150: 5 \\
1274 \\
1127 \\
1283 \\
1114\end{array}$ & 5187 & $\begin{array}{l}46,0 \\
56,5 \\
59,8 \\
68,2 \\
55,2\end{array}$ & 230,5 & $\begin{array}{l}1,03 \\
1,48 \\
1,77 \\
1,77 \\
1,65\end{array}$ \\
\hline 15 & & 970 & 6301 & - & 285,7 & - & 1,51 \\
\hline
\end{tabular}

V. 20. Oktober 1886.

\begin{tabular}{|c|c|c|c|c|c|c|c|}
\hline Zahl & $\begin{array}{c}\text { Tages- } \\
\text { zeit } \\
\text { mg }\end{array}$ & $\begin{array}{l}\text { Harn- } \\
\text { menge } \\
\mathrm{ccm}\end{array}$ & $\begin{array}{c}\text { Harn- } \\
\text { stickstoff } \\
\text { mg }\end{array}$ & $\begin{array}{c}\text { Gesamt- } \\
\text { menge in } \\
12 \mathrm{Stdn} . \\
\mathrm{mg}\end{array}$ & $\begin{array}{c}\text { Harn- } \\
\text { säure } \\
\text { mg }\end{array}$ & $\begin{array}{c}\text { Gesamt- } \\
\text { menge in } \\
12 \text { Stdn. } \\
\mathrm{mg}\end{array}$ & $\begin{array}{c}\text { Anteil der } \\
\text { Harnsäure } \\
\text { a. Gesamt- } \\
\text { stickstoff } \\
\%\end{array}$ \\
\hline $\begin{array}{l}1 . \\
2 . \\
3 . \\
4 . \\
5 .\end{array}$ & $\begin{array}{c}6-9 \\
9-12 \\
12-3 \\
3-6 \\
6-9\end{array}$ & $\begin{array}{r}230 \\
256 \\
204 \\
212 \\
90\end{array}$ & $\begin{array}{l}1998 \\
1796 \\
1448 \\
1092 \\
1006\end{array}$ & 6634 & $\begin{array}{l}66,7 \\
54,3 \\
46,3 \\
47,5 \\
28,6\end{array}$ & $\mathbf{2 1 4}, 8$ & $\begin{array}{l}1,11 \\
1,00 \\
1,06 \\
1,13 \\
0,84\end{array}$ \\
\hline 15 & & 992 & 7640 & - & 243,4 & - & 1,06 \\
\hline
\end{tabular}

VI. 3. Norember $18 \& 6$.

\begin{tabular}{|c|c|c|c|c|c|c|c|}
\hline Zahl & $\begin{array}{l}\text { Tages- } \\
\text { zeit } \\
\text { Uhr }\end{array}$ & $\begin{array}{l}\text { Harn- } \\
\text { menge } \\
\text { ccm }\end{array}$ & $\begin{array}{c}\text { Harn- } \\
\text { stickstoff } \\
\text { mg }\end{array}$ & $\begin{array}{c}\text { Gesamt- } \\
\text { menge in } \\
12 \mathrm{Stdn} . \\
\mathrm{mg}\end{array}$ & $\begin{array}{c}\text { Harn- } \\
\text { säure } \\
\text { mg }\end{array}$ & $\begin{array}{c}\text { Gesamt- } \\
\text { menge in } \\
12 \text { Stdn. } \\
\text { mg }\end{array}$ & $\begin{array}{c}\text { Auteil der } \\
\text { Harnsäure } \\
\text { a. Gesamt- } \\
\text { stickstoff } \\
\%\end{array}$ \\
\hline $\begin{array}{l}1 . \\
2 . \\
3 . \\
4 . \\
5 .\end{array}$ & $\begin{array}{c}6-9 \\
9-12 \\
12-3 \\
3-6 \\
6-6\end{array}$ & $\begin{array}{r}230 \\
150 \\
106 \\
102 \\
74\end{array}$ & $\begin{array}{l}2436 \\
1768 \\
1519 \\
1466 \\
1274\end{array}$ & 7189 & $\begin{array}{l}85,1 \\
50,7 \\
60,4 \\
50,1 \\
44,5\end{array}$ & 246,3 & $\begin{array}{l}1,16 \\
0,95 \\
1,32 \\
1,14 \\
1,16\end{array}$ \\
\hline n 15 & $n$ & 662 & 8463 & - & 290,8 & - & 1,14 \\
\hline
\end{tabular}


Ein experim. Beitrag zur Lebre von der individuellen Konstanz etc. 537

VII. 16. November 1886.

\begin{tabular}{|c|c|c|c|c|c|c|c|}
\hline Zahl & $\begin{array}{l}\text { Tages- } \\
\text { zeit } \\
\text { Uhr }\end{array}$ & $\begin{array}{c}\text { Harn- } \\
\text { menge } \\
\text { ccm }\end{array}$ & $\begin{array}{c}\text { Harn- } \\
\text { stickstoff } \\
\text { mg }\end{array}$ & $\begin{array}{l}\text { Gesamt- } \\
\text { menge in } \\
12 \text { Stdn. } \\
\text { mg }\end{array}$ & $\begin{array}{c}\text { Harn- } \\
\text { säure } \\
\text { mg }\end{array}$ & $\begin{array}{c}\text { Gesamt- } \\
\text { menge in } \\
12 \text { Stdn. } \\
\text { mg }\end{array}$ & $\begin{array}{c}\text { Anteil der } \\
\text { Harnsäure } \\
\text { a. Gesamt- } \\
\text { stickstoff } \\
\quad / 0\end{array}$ \\
\hline $\begin{array}{l}1 . \\
2 . \\
3 . \\
4 . \\
5 .\end{array}$ & $\begin{array}{c}6-9 \\
9-12 \\
12-3 \\
3-6 \\
6-9\end{array}$ & $\begin{array}{r}210 \\
147 \\
100 \\
74 \\
62\end{array}$ & $\begin{array}{r}1712 \\
1377 \\
1176 \\
900 \\
940\end{array}$ & 5165 & $\begin{array}{l}78,5 \\
58,7 \\
52,7 \\
25,2 \\
46,4\end{array}$ & 215,1 & $\begin{array}{l}1,52 \\
1,42 \\
1,49 \\
0,93 \\
1,64\end{array}$ \\
\hline 15 & n & 593 & 6105 & - & 261,5 & - & 1,42 \\
\hline
\end{tabular}

VIII. 93. November $18 \& 6$.

\begin{tabular}{|c|c|c|c|c|c|c|c|}
\hline Zahl & $\begin{array}{l}\text { Tages- } \\
\text { zeit } \\
\text { Uhr }\end{array}$ & $\begin{array}{l}\text { Harn- } \\
\text { menge } \\
\text { ccm }\end{array}$ & $\begin{array}{c}\text { Harn- } \\
\text { stickst off } \\
\text { mg }\end{array}$ & $\begin{array}{c}\text { Gesamt- } \\
\text { menge in } \\
12 \text { Stdn. } \\
\text { mg }\end{array}$ & $\begin{array}{l}\text { Harn- } \\
\text { säure } \\
\text { mg }\end{array}$ & $\begin{array}{l}\text { Gesamt- } \\
\text { menge in } \\
12 \text { Stdn. } \\
\text { mg }\end{array}$ & $\begin{array}{c}\text { Anteil der } \\
\text { Harnsäure } \\
\text { a. Gesamt- } \\
\text { stickstoff } \\
\% \\
\%\end{array}$ \\
\hline $\begin{array}{l}1 . \\
2 . \\
3 \\
4 . \\
5 .\end{array}$ & $\begin{array}{c}6-9 \\
9-12 \\
12-3 \\
3-6 \\
6-9\end{array}$ & $\begin{array}{r}276 \\
242 \\
226 \\
160 \\
90\end{array}$ & $\begin{array}{l}2658 \\
2127 \\
1833 \\
1536 \\
1278\end{array}$ & 8154 & $\begin{array}{l}71,8 \\
71,2 \\
61,0 \\
45,8 \\
39,9\end{array}$ & 249,8 & $\begin{array}{l}0,89 \\
1,11 \\
1,10 \\
0,99 \\
1,04\end{array}$ \\
\hline 15 & en & 994 & 9432 & & 289,7 & & 1,02 \\
\hline
\end{tabular}

IX. 30. Norember 1986 .

\begin{tabular}{|c|c|c|c|c|c|c|c|}
\hline Zahl & $\begin{array}{c}\text { Tages- } \\
\text { zeit } \\
\text { Uhr }\end{array}$ & $\begin{array}{l}\text { Harn- } \\
\text { menge } \\
\text { ccm }\end{array}$ & $\begin{array}{c}\begin{array}{c}\text { Harn- } \\
\text { stickstoff }\end{array} \\
\text { mg }\end{array}$ & $\begin{array}{c}\text { Gesamt- } \\
\text { menge in } \\
12 \text { Stdn. } \\
\mathrm{mg}\end{array}$ & $\begin{array}{l}\text { Harn- } \\
\text { säure } \\
\text { mg }\end{array}$ & $\begin{array}{l}\text { Gesamt- } \\
\text { menge in } \\
12 \text { Stdn. } \\
\text { mg }\end{array}$ & $\begin{array}{c}\text { Anteil des } \\
\text { Harnsäure } \\
\text { a. Gesamt- } \\
\text { stickstoff } \\
\quad \%\end{array}$ \\
\hline $\begin{array}{l}1 . \\
2 . \\
3 . \\
4 . \\
5 .\end{array}$ & $\begin{array}{c}6-9 \\
9-12 \\
12-3 \\
3-6 \\
6-9\end{array}$ & $\begin{array}{r}150 \\
90 \\
86 \\
82 \\
66\end{array}$ & $\begin{array}{l}2169 \\
1602 \\
1559 \\
1438 \\
1300\end{array}$ & 6768 & $\begin{array}{l}64,8 \\
59,1 \\
57,9 \\
49,5 \\
44,1\end{array}$ & 281,3 & $\begin{array}{l}0,99 \\
1,22 \\
1,23 \\
1,14 \\
1,13 \\
\end{array}$ \\
\hline \multicolumn{2}{|c|}{ In 15 Stunden } & \multicolumn{6}{|c|}{ X. 6. Dezember 1986 . } \\
\hline Zahl & $\begin{array}{c}\text { 'Tages- } \\
\text { zeit } \\
\text { Uhr }\end{array}$ & $\begin{array}{l}\text { Harn- } \\
\text { menge } \\
\text { ccm }\end{array}$ & $\begin{array}{c}\text { Harn- } \\
\text { stickstoff } \\
\text { mg }\end{array}$ & $\begin{array}{c}\text { Gesamt- } \\
\text { menge in } \\
12 \text { Stdn. } \\
\text { mg }\end{array}$ & $\begin{array}{c}\text { Harn- } \\
\text { säure } \\
\text { mg }\end{array}$ & $\begin{array}{c}\text { Gesamt- } \\
\text { menge in } \\
12 \text { Stdn. } \\
\text { mg }\end{array}$ & $\begin{array}{c}\text { A nteil der } \\
\text { Harnsäure } \\
\text { a. Gesamt- } \\
\text { stickstoff } \\
\% \\
\end{array}$ \\
\hline $\begin{array}{l}1 . \\
2 . \\
3 . \\
4 . \\
5 .\end{array}$ & $\begin{array}{c}6-9 \\
9-12 \\
12-3 \\
3-6 \\
6-9\end{array}$ & $\begin{array}{r}150 \\
94 \\
82 \\
74 \\
59\end{array}$ & $\begin{array}{l}1777 \\
1324 \\
1247 \\
1157 \\
1038\end{array}$ & 5505 & $\begin{array}{l}65,9 \\
52,8 \\
50,1 \\
41,8 \\
30.0\end{array}$ & 210,6 & $\begin{array}{l}1,28 \\
1,33 \\
1,35 \\
1,20 \\
0,96\end{array}$ \\
\hline n 15 & & 459 & 6543 & & 240,6 & & 1,29 \\
\hline
\end{tabular}


XI. 14. Dezember 1886 .

\begin{tabular}{|c|c|c|c|c|c|c|c|}
\hline Zahl! & $\begin{array}{l}\text { Tages- } \\
\text { zeit } \\
\text { Ohr }\end{array}$ & $\begin{array}{l}\text { Harn- } \\
\text { menge } \\
\text { ccm }\end{array}$ & $\begin{array}{c}\text { Harn- } \\
\text { stickstoff } \\
\text { mg }\end{array}$ & $\begin{array}{c}\text { Gesamt- } \\
\text { menge in } \\
12 \text { Stdn. } \\
\text { mg }\end{array}$ & $\begin{array}{l}\text { Harn- } \\
\text { säure } \\
\text { mg }\end{array}$ & $\begin{array}{c}\text { Gesamt- } \\
\text { menge in } \\
12 \text { Stdn. } \\
\mathrm{mg}\end{array}$ & $\begin{array}{c}\text { Anteil der } \\
\text { Harnsäure } \\
\text { a. Gesamt- } \\
\text { stickstoff } \\
0 \%\end{array}$ \\
\hline $\begin{array}{l}1 . \\
2 . \\
3 . \\
4 . \\
5 .\end{array}$ & $\begin{array}{c}6-9 \\
9-12 \\
12-3 \\
3-6 \\
6-9 \\
\end{array}$ & $\begin{array}{r}260 \\
214 \\
148 \\
90 \\
64 \\
\end{array}$ & $\begin{array}{l}3086 \\
2350 \\
1857 \\
1522 \\
1240 \\
\end{array}$ & 8815 & $\begin{array}{l}76,4 \\
61,9 \\
46,5 \\
48,9 \\
39,9 \\
\end{array}$ & 293,7 & $\begin{array}{l}0,82 \\
0,88 \\
0,83 \\
1,07 \\
1,06 \\
\end{array}$ \\
\hline 15 & en & 776 & 10055 & - & 273,6 & - & 0,90 \\
\hline
\end{tabular}

Übersichtstabelle der neun Tagesversuche an der Person A rom Jahre 1886, mit Beschränkung anf die zwölfstündigen Gesamtwerte der Harnsäure.

\begin{tabular}{|c|c|c|c|}
\hline $\begin{array}{c}\text { Datum } \\
\text { des } \\
\text { Versuches }\end{array}$ & $\begin{array}{c}\text { Nummer } \\
\text { des } \\
\text { Versuches }\end{array}$ & $\begin{array}{c}\text { Tageszeit } \\
\text { Uhr }\end{array}$ & $\begin{array}{c}\text { Harnsäure- } \\
\text { menge für } \\
12^{\circ} \text { Stunden } \\
\text { mg }\end{array}$ \\
\hline $\begin{array}{l}\text { 16. Juni } \\
22 . \text { " } \\
20 \text {. Oktober } \\
\text { 3. November } \\
\text { 16. " " } \\
23 . \text { " } \\
30 . \text { " } \\
\text { 6. Dezember } \\
\text { 14. " }\end{array}$ & $\begin{array}{l}\text { I } \\
\text { II } \\
\text { V } \\
\text { VI } \\
\text { VII } \\
\text { VIII } \\
\text { IX } \\
\text { X } \\
\text { XI }\end{array}$ & $\left\{\begin{array}{c}\text { Immer von } \\
6 \mathrm{Uhr} \text { früh } \\
\text { bis } \\
6 \text { Uhr abends }\end{array}\right.$ & $\begin{array}{l}247,9 \\
230,5 \\
214,8 \\
246,3 \\
215,1 \\
249,8 \\
231,3 \\
210,6 \\
233,7\end{array}$ \\
\hline
\end{tabular}

In neun Versuchen zu 12 Stunden zusammen $2080,0 \mathrm{mg}$ Harnsäure ansgeschieden (: 9). In einem Versuche, oder: für 12 Stunden

durchschnittlich . . . . . . 231,1 $" \quad \# \quad \#$ (: 12).

In 1 Stunde durchschnittlich . . 19,2 " "

$\left.\begin{array}{c}\text { In } 24 \text { Stunden durchschnittlich für } \\ 1 \mathrm{~kg} \text { Körpergewicht . . . . }\end{array}\right\} 8,11, \quad "$

Über die Versuche aus dem Jabre 1911, die jetzt folgen werden, sei noch bemerkt, dass der Harn auch in dreistündigen Pbasen gesammelt und analysiert wurde. Die Bestimmungen der Harnsäure geschahen nach Salkowski-Ludwig, die des Gesamtstickstoffes nach Kjeldahl, konform mit den Versuchen aus dem Jahre 1886.

Die Versuchsperson A, Staatsbediensteter J. K., fühlt sich und ist in seinem 70. Lebensjahre noch gesund, hat entsprechenden Appetit, wobei ihm sein noch vollständiges, musterhaftes Gebiss gute Dienste leistet, lebt regelmässig, ist entsprechend arbeitsfähig. 
Ein experim. Beitrag zur Lehre von der individuellen Konstanz etc. 539

\section{Versuche vom Jahre 1911.}

Versuch 1. 29. April 1911.

Mann A, 70 Jahre, Körpergewicht $57 \mathrm{~kg} 350 \mathrm{~g}$, Körperlänge $157 \mathrm{~cm}$.

An dem Tage vor dem Versuche (28. April) Frühstück (1 Tasse weissen Kaffee, 1 Kipfel) um $6^{1 / 2}$ Uhr früh. Um 11 Uhr Mittagessen, bestehend aus Kartoffelsuppe (ohne Rindsuppe) und Sterz. Nachtmahl um 5 Uhr 20 Min., Kartoffelsuppe und 243,3 g weichen Topfens und genug Trinkwasser. Um 5 Uhr an demselben Vorversuchstage Blasenentleerung, um 6 Uhr dasselbe in die Mensur, wobei die Analyse ausfällt:

28. April 1911, $5-6$ Uhr nachm., Harnmenge $136 \mathrm{ccm}$, Gesamtstickstoff $441,7 \mathrm{mg}$.

Anfang des Versuches am 29. April durch Blasenentleerung um 6 Uhr früh.

\begin{tabular}{|c|c|c|c|c|c|}
\hline Zahl & $\begin{array}{c}\text { Tageszeit } \\
\text { Uhr }\end{array}$ & $\begin{array}{c}\text { Harnmenge } \\
\text { ccm }\end{array}$ & $\begin{array}{c}\text { Harn- } \\
\text { stickstoff } \\
\text { mg }\end{array}$ & $\begin{array}{l}\text { Harnsäure } \\
\text { mg }\end{array}$ & $\begin{array}{c}\text { Anteil der } \\
\text { Harnsäure } \\
\text { am Gesamt- } \\
\text { stickstoff } \\
0 / 0\end{array}$ \\
\hline $\begin{array}{l}1 . \\
2 . \\
3 . \\
4 .\end{array}$ & $\begin{array}{c}6-9 \\
9-12 \\
12-3 \\
3-6\end{array}$ & $\begin{array}{l}175 \\
158 \\
130 \\
104\end{array}$ & $\begin{array}{l}1449,7 \\
1336,2 \\
1179,3 \\
1056,15\end{array}$ & $\begin{array}{l}63,21 \\
62,88 \\
59,69 \\
51,45\end{array}$ & $\begin{array}{l}1,47 \\
1,57 \\
1,69 \\
1,62\end{array}$ \\
\hline $12 \mathrm{St}$ & ${ }^{-}$ & 567 & 5021,35 & 287,23 & 1,57 \\
\hline
\end{tabular}

Versuch 2. 19. Mai 1911.

Mann A, 70 Jahre, Körpergewicht $57 \mathrm{~kg}, 260 \mathrm{~g}$, Körperlänge $157 \mathrm{~cm}$.

Am Vorversuchstage um $6^{1 / 2}$ Uhr weissen Kaffee und 1 Kipfel gefrühstückt. Mittagmahl um 11 Uhr: Kartoffelsuppe, Knödeln mit Butter. Nachtmahl um 5 Uhr 35 Min.: Kartoffelsuppe, um 5 Uhr 50 Min. hat er das feinste, fettlose, gekochte Rindfleisch zu essen angefangen. Bis 5 Uhr 59 Min. hat er sich mit $130,85 \mathrm{~g}$ desselben und mit $47,85 \mathrm{~g}$ Semmel gesättigt. Genug Trinkwasser. Um 9 Uhr abends zwei Achtel Krondorfer. Um 3 Uhr nachmittags am: selben Versuchstage Harn gelassen. Um 6 Uhr ebenso in die Mensur; Analyse dieser Phase ergibt: 
18. Mai 1911, 3- ל’ nachm., Harnmenge $147,5 \mathrm{ccm}$, Gesamtstickstoff $1483,03 \mathrm{mg}$.

Anfang des Versuches am 19. Mai 1911 um 6 Uhr früh durch Blasenentleerung.

\begin{tabular}{c|c|c|c|c|c}
\hline \hline Zahl & Tageszeit & Harnmenge & $\begin{array}{c}\text { Harn- } \\
\text { stickstoff }\end{array}$ & Harnsäure & $\begin{array}{c}\text { Anteil der } \\
\text { Harnsäure } \\
\text { am Gesamt- } \\
\text { stickstoff } \\
\%\end{array}$ \\
\hline Uhr & ccm & $\mathrm{mg}$ & $\mathrm{mg}$ & $\%$ \\
\hline 1. & $6-9$ & 152,5 & 1570,50 & 65,62 & 1,39 \\
2. & $9-12$ & 147,5 & 1444,45 & 62,23 & 1,43 \\
3. & $12-3$ & 157,5 & 1446,19 & 583 & 1,34 \\
4. & $3-6$ & 122,5 & 1228,22 & 46,95 & 1,27 \\
\hline In 12 Stunden . . . . & 580,0 & 5689,36 & $\mathbf{2 3 3 , 1 0}$ & 1,37
\end{tabular}

In 1 Stunde durchschnittlich . . . . . . . . . . . 19,7

In 24 Stund en für $1 \mathrm{~kg}$ Körpergewicht . . . . . . . 8,27

Übersichtstabelle der zwei Tagesversuche an der Person A rom Jahre 1911, mit zwölfstündigen Gesamtwerten der Harnsäure.

\begin{tabular}{|c|c|c|c|}
\hline $\begin{array}{c}\text { Datum } \\
\text { des } \\
\text { Versuches }\end{array}$ & $\begin{array}{c}\text { Nummer } \\
\text { des } \\
\text { Versuches }\end{array}$ & $\begin{array}{c}\text { Tageszeit } \\
\text { Uhr }\end{array}$ & $\begin{array}{l}\text { Harnsäure- } \\
\text { menge in } \\
12 \text { Stunden } \\
\text { mg }\end{array}$ \\
\hline $\begin{array}{l}\text { 29. April } \\
\text { 19. Mai }\end{array}$ & $\begin{array}{l}1 \\
2\end{array}$ & $\left\{\begin{array}{c}\text { Immer von } 6 \text { Uhr früh } \\
\text { bis } 6 \text { Uhr abends }\end{array}\right.$ & $\begin{array}{l}237,23 \\
239,10\end{array}$ \\
\hline
\end{tabular}

In zwei Versuchen zu 12 Stunden zusammen 470,33 mg Harnsäure ausgeschieden (: 2).

In einem Versuche oder für 12 Stunden

durchschnittlich . . . . . . 285, 16 "

In 1 Stunde durchschnittlich. . . . . . 19,59,

In 24 Stunden für $1 \mathrm{~kg}$ Körpergewicht . 8,2 ,

Wenn wir nun kurz rekapitulieren, sehen wir:

Im Jahre 1886 zählte die Versuchsperson, Mann A . 45 Jabre er hatte die Körperlänge. . . . . . . . . . $157 \mathrm{~cm}$ er hatte das Körpergewicht von . . . . . . . $57 \mathrm{~kg}$ er schied während des Tages der Harnsäure aus:

in 12 Stunden im Mittel . . . . . . . . 281,1 mg

in 1 Stunde im Mittel . . . . . . . . . 19,2 "

in 24 Stunden für $1 \mathrm{~kg}$ Körpergewicht . . . 8,11 n

Im Jahre 1911 zählte die Versuchsperson, Mann A . 70 Jahre seine Körperlänge war . . . . . . . . . . $157 \mathrm{~cm}$ sein Körpergewicht war im Mittel . . . . . . 57,305 kg 
Ein experim. Beitrag zur Lehre von der individuellen Konstanz etc. 541

er schied während des Tages der Harnsäure aus:

in 12 Stunden im Mittel . . . . . . . 2855,16 mg

in 1 Stunde im Mittel . . . . . . . . . 19,59 "

in 24 Stunden für $1 \mathrm{~kg}$ Körpergewicht . . . 8,2

Das Resultat der neuen Versuchsreihe gestaltete sich somit mit einer unerwarteten Vollkommenheit zugunsten der Lehre von der individuellen Konstanz dex in einer bestimmten Periode des nüchternen Zustandes ausgeschiedenen Harnsäuremenge, Es ist wirklich ein erstaunliches Dokument der Zähigkeit, mit der der menschliche Organismus an seiner individuellen Menge der ausgeschiedenen Harnsäure festhält, wenn hier eine und dieselbe Versuchsperson A nach fünfundzwanzig Jahren unter sonst gleichen Bedingungen genau dieselbe Menge der Harnsäure liefert wie zuvor. Die Lehre von der individuellen Harnsäurekonstanz wird hier um eine in der diesbezüglichen physiologischen Literatur einzige Angabe von der Identität der im nüchternen Zustande ausgeschiedenen Harnsäuremenge vom Mannesalter durch volle fünfundzwanzig Jahre bis in das Greisenalter bereichert. Die erste tatsächliche Grundlage der von Mareš herrührenden Theorie über die Herkunft der Harnsäure beim Mensehen erfährt durch dieses Resultat an Festigkeit. Und wenn wir uns erinnern, wie Folin ${ }^{1}$ ) für die Annahme, dass die endogene Harnsäure ein exklusives Derivat der Zellnukleine ist oder, im Wortlaute der Theorie von Mare ̌̌, dass die Harnsäure ein Produkt des physiologischen Stoffwechsels im Protoplasma der Körperzellen (wobei namentlich die Nukleine der Zellkerne beteiligt sind), einen genau en $\mathrm{Nachweis} \mathrm{der} \mathrm{individuellen} \mathrm{Konstanz} \mathrm{der}$ Ausseheidung des endogenen Harnsäurewertes als eine starke Stütze bezeichnet und verlangt, so glaube ich durch das Resultat dieser meinen Untersuchungen $z u$ einem solchen Nachweis beigetragen zu haben.

Das Ergebnis dieser Untersuchungen bildet auch einen auf einwandfreie Weise gewonnenen Beitrag zu der Frage über das Verhalten der Harnsäureausscheidung im physiologischen Zustande der Nüchternheit wahrend des Greisenalters. Wenn wir das Greisentum als eine Abschwächung der physiologischen Funktionen auffassen, so können wir auf Grund der vorhergehenden Versuchsergebnisse vielleicht doch den $Z$ weifel aussprechen, ob sich das Altern

1) Folin, The Americ. Journ. of Physiol. vol. 13 p. 66. 1905. 
auch auf die Produktion und Ausscheidung der Harnsäure bezieht? Wird da der Organismus alt? Oder bleibt er in dieser Funktion stationär? Nur noch weitere Untersuchungen können da Aufschluss geben.

Ich ging noch weiter daran, das Material der Lehre von der individuellen Konstanz der Harnsäureausscheidung durch einen neuen Beleg zu erweitern, und zwar auf Grund einer anderen Erwägung und in einer etwas geänderten Versuchsanordnung. Ich habe oben angeführt, dass die Mehrzahl der Autoren auch bei abermaliger Darreichung der Nahrung unter fixer Lebensweise, so wie dies im Stickstoffgleichgewicht der Fall ist, die Tatsache der Harnsäurekonstanz bestätigte, und ich erwähnte, dass $\mathrm{S}$ metán $\mathrm{ka}^{1}$ ) gelegentlich seiner zwei zur Feststellung des Einflusses der Verdạungstätigkeit bei purinfreier Nahrung an einem und demselben Individuum im nüchternen Zustande bei einmaliger Nahrungsaufnahme im Zeitraume von weniger als zwei Monaten angestellten Versuche auf dasselbe aufmerksam machte. Ich versuchte nun an einer Versuchsperson aus seiner Versuchsreihe, die sich noch dazu bereit zeigte, Ähnliches, aber nach einer geraumen Zeit, nach mehr als drei und einem halben Jahre.

Man kann den Zustand eines ständig in gleichen Verhältnissen lebenden Organismus als einen stationären bezeichnen. Diesen $\mathrm{Zu}$ stand stört man, wenn man bestimmte physiologische Verrichtungen eines solchen Organismus durch Reizung erhöht oder wenn man bestimmte physiologische Verrichtungen erniedrigt oder ausschaltet. Der nüchterne Zustand ist eben eine Änderung des stationären in dem letzterwähnten Sinne: der Verdauungsapparat; besonders seine Drüsen, ruht, die herabgesetzten Stoffwechselvorgänge verlaufen noch regelmässiger, die Menge der gleichzeitig ausgeschiedenen Harnsäure stabilisiert sich an einem verminderten konstanten Niveau. Wie oben gezeigt, ist es selbst nach einer geraumen Zeit möglich, denselben Organismus unter Anwendung der Methode des nüchternen Zustandes in denselben Zustand der Gleichmässigkeit in den herabgesetzten Stoffwechselvorgängen zu versetzen und dasselbe konstante Maass der ausgeschiedenen Harnsäure zu erhalten.

1) F. Smetánka, Żur Herkunft der Harnsäure beim Menschen. Arch. f. d. ges. Physiol. Bd. 138 S. 226, 229, 267. 1911. 
Ein experim. Beitrag zur Lehre von der individuellen Konstanz etc. 543

Werden sich aber nach einem grossen Zeitabstand bei demselben in den Zustand der Nüchternheit versetzten Organismus durch den gleichen einmaligen Reiz die betreffenden Verrichtungen in gleicher Weise wie zuvor erregen lassen? Wird sich nach demselben Reiz dieselbe Reaktion in derselben Proportionalität einstellen?

Wenn ich an der in Rede stehenden Person, an welcher im Jahre 1909 ein Versuch im nüchternen Zustande mit einmaliger Darreichung einer purinfreien Nahrung ausgeführt wurde, genau denselben Versuch unter genau denselben Verhältnissen unternehmen werde: wird es gelingen, durch die einmalige Anregung des nüchternen Verdauungsapparates, seiner Drüsen, auch die Vermehrung der Produktion der Harnsäure über das dem nüchternen Zustande entsprechende wohl als konstant zu erwartende Niveau in demselben Maasse wie einst zu erzielen? Wird sich auch hier eine diesbezügliche Konstante herausstellen?

Die Versuchsperson Herr E. S. ist ein gesunder Mann, der sich aus Interesse dem Versuche unterwirft.

Es folgt nun zuerst der betreffende Versuch Smetánka's').

\section{Versuch III.}

2. November 1909. E. S., Ph.-Dr., Universitätsprofessor, 34 Jahre, $92 \mathrm{~kg}$. Letzte Nahrungsaufnahme (purinfreie Nahrung) 18 Stunden vor Beginn des Versuches. Am Versuchstage um 6 Uhr aufgestanden, zwischen 9 Uhr 5 Min. und 9 Uhr 15 Min. $203 \mathrm{~g}$ To $\mathrm{p}$ f e $\mathrm{n}$ genossen, etwa $250 \mathrm{ccm}$ Wasser getrunken.

\begin{tabular}{|c|c|c|c|}
\hline Zahl & $\begin{array}{c}\text { Tageszeit } \\
\text { Uhr }\end{array}$ & $\begin{array}{c}\text { Harnmenge } \\
\text { ccm }\end{array}$ & $\begin{array}{c}\text { Harnsäure } \\
\text { mg }\end{array}$ \\
\hline 1. & $\begin{array}{l}7-8 \mathrm{~h} \text { morgens } \\
8-9 \mathrm{~h}\end{array}$ & $\begin{array}{l}39,5 \\
48,0\end{array}$ & $\begin{array}{l}31,4 \\
31,4\end{array}$ \\
\hline $\begin{array}{l}3 . \\
4 . \\
5 . \\
6 . \\
7 . \\
8 . \\
9 .\end{array}$ & $\begin{array}{l}9-10 \mathrm{~h} \\
10-11 \mathrm{~h} \quad " \\
11-12 \mathrm{~h} \text { mittags } \\
12-1 \mathrm{~h} \text { nachmittags } \\
1-2 \mathrm{~h} \quad " \\
2-3 \mathrm{~h} \quad " \\
3-4 \mathrm{~h} "\end{array}$ & $\begin{array}{l}51,5 \\
58,0 \\
91,0 \\
75,0 \\
59,0 \\
67,0 \\
78,0\end{array}$ & $\begin{array}{l}40,8 \\
54,7 \\
56,2 \\
34,5 \\
25,8 \\
27,1 \\
29,1\end{array}$ \\
\hline Stu & & 567,0 & 391,0 \\
\hline
\end{tabular}

1) F. Smetánka, Zur Herkunft der Harnsäure beim Menschen. Arch. f. d. ges. Physiol. Bd. 134 S. 227. 1911. 
Es folgt weiter mein

\section{Versuch 3.}

5. Juli 1913. E. S., Ph.-Dr., Universitätsprofessor, 38 Jahre, Körpergewicht $96,5 \mathrm{~kg}$. Am Tage zuvor (4. Juli 1913) letzte Nahrungsaufnahme in der Form eines purinfreien Mittagsmahles (Einbrennsuppe mit Reis und einem gerührten Ei, Knödeln mit Dillensauce, etwas Trinkwasser) zwischen $1-11 / 4$ Uhr; um 7 Uhr abends eine kleine Flasche Krondorfer. Am Versuchstage um 6 Uhr wach geworden, um 6 Uhr 15 Min. aufgestanden; um 7 Uhr 15 Min. erste Blasenentleerung. Beginn des Versuches geschah also wieder 18 Stunden nach der letzten purinfreien Nahrungsaufnahme. Zwischen 9 Uhr 17 Min. und 9 Uhr 30 Min. $211 \mathrm{~g}$ Topfen zu sich genommen, etwa $250 \mathrm{ccm}$ Krondorfer ausgetrunken.

\begin{tabular}{|c|c|c|c|}
\hline Zahl & $\begin{array}{c}\text { Tageszeit } \\
\text { Uhr }\end{array}$ & $\begin{array}{c}\text { Harnmenge } \\
\mathrm{ccm}\end{array}$ & $\begin{array}{c}\text { Harnsäure } \\
\text { mg }\end{array}$ \\
\hline $\begin{array}{l}1 . \\
2 .\end{array}$ & $\begin{array}{l}7^{1 / 4}-8^{1 / 4} \text { h h morgens } \\
8^{1 / 4}-9^{1 / 4} \text { h }\end{array}$ & $\begin{array}{l}51 \\
49\end{array}$ & $\begin{array}{l}32,9 \\
29,5\end{array}$ \\
\hline $\begin{array}{l}3 . \\
4 . \\
5 . \\
6 . \\
7 . \\
8 . \\
9 .\end{array}$ & 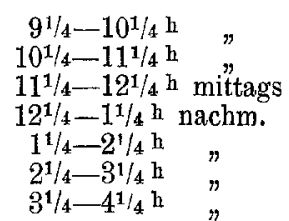 & $\begin{array}{l}54 \\
70 \\
81 \\
91 \\
61 \\
82 \\
69\end{array}$ & $\begin{array}{l}32,3 \\
46,8 \\
50,9 \\
38,9 \\
34,0 \\
26,5 \\
22,9\end{array}$ \\
\hline$\overline{\text { Stu }}$ & 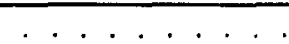 & 608 & 314,7 \\
\hline
\end{tabular}

Die Einzelheiten der Lebensweise und der Diät, die im Versuche III nicht erwähnt sind, wurden aus dem Häuslichkeitstagebuche der Versuchsperson eruiert und im Versuche 3 genau verwirklicht.

Wenn wir die Resultate der beiden Versuche überblicken, so finden wir:

Im Jahre 1909 zählte die Versuchsperson Herr E. S. . 34 Jahre

er wies auf das Körpergewicht von . . . . . . $92 \mathrm{~kg}$

er schied während des Tages in 9 Stunden des nüchternen Zustandes mit einmaliger purinfreier Nahrungsaufnabme der Harnsäure aus . . . . 331,0 mg. Im Jahre 1913 zählte dieselbe Person, Herr E. S. . . 38 Jahre sein Körpergewicht vergrösserte sich auf . . . . . $96,5 \mathrm{~kg}$

Die Ausscheidung der Harnsäure während des Tages in 9 Stunden des nüchternen Zustandes unter einmaliger purinfreier Nahrungsaufnahme beträgt $\mathbf{3 1 4 , 7} \mathrm{mg}$. 
Ein experim. Beitrag zur Lehre von der individuellen Konstanz etc. 545

Auch unter solchen sonst gleichen Versuchsbedingungen, nach einem Zeitabstand von beinahe $4 \mathrm{Jahr}$ en erweist sich die Gesamtmenge der ausgeschiedenen Harnsäure auch nach einmaliger purinfreier Nahrungszufuhr als eine individuelle Konstante, indem sie im Versuche III $331 \mathrm{mg}$ in 9 Stunden, im Versuche 3 für die gleichen 9 Stunden $314,7 \mathrm{mg}$, also eine $\mathrm{n}$ ahezu gleiche, im Versuche 3 nur um etwa $4 \%$ kleinere Menge ausmacht. Die Harnsäurevermehrung nach der Nahrungsaufnahme zeigt zwar in dem Versuche 3 eine Abweichung von derjenigen im Versuche III; sie setzt etwas später ein, erreicht nicht absolut dieselbe Höhe, währt dafür länger, um dann ähnlich wie im Versuche III abzufallen, wie dies aus dem beigefügten Diagramme ersichtlich ist. Die Reaktion auf die Anregung der Verdauungsdrüsentätigkeit gestaltete sich in ihrem zeitlichen Verlaufe diesmal etwas träger. Diese Verlangsamung der Ausscheidung könnte man als eine Änderung der Individualität des betreffenden Drüsenapparates deuten. Man könnte auch fragen, ob diesmal auch der psychische Reiz, der Appetit, in dem Maasse mitwirkte wie im Versuche III? Abgesehen davon, liefert der Versuch 3 noch eine Bestätigung der Befunde Smetánka's über den die Harnsäureausscheidung vermehrenden Einfluss der purinfreien Nahrung.

Ich resumiere nun folgendermaassen:

Auf Grund der Versuche von Mares, welcher zuerst an denselben Individuen in Zeitabständen von 6 Monaten, von 1 Monate im nüchternen Zustande unter gleichen Verhältnissen dieselben Harnsäurewerte fand und diese zuerst als Harnsäurekonstante (constante d'acide urique) bezeichnete, was eine von den Grundlagen seiner Theorie des Ursprungs der Harnsäure beim Menschen bildet,

auf Grund der Versuche Burian's und Schur's, die an einem Subjekte im Stickstoffgleichg e w i cht dieselbe Menge der ausgeschiedenen Harnsäure nach einem Zeitspatium von $5 \mathrm{Mo}$.

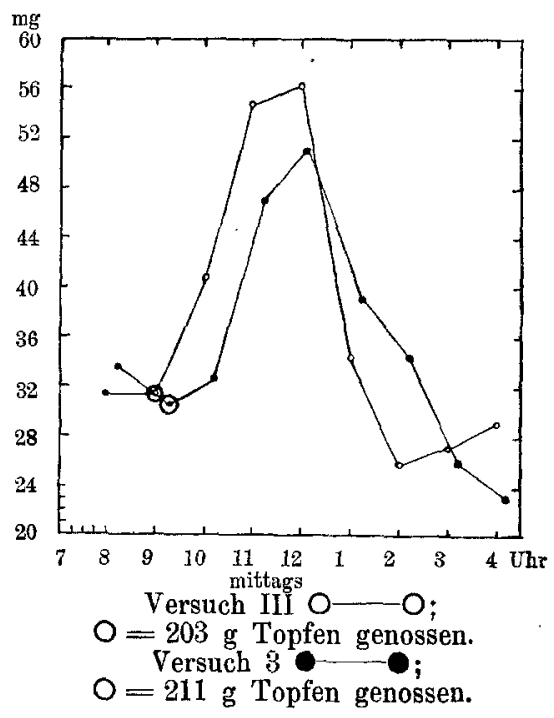


546 Ot. Faustka: Ein experim. Beitrag zur Lehre von der indiv. Konstanz etc.

naten konstatierten und sie für eine physiologische Konstante hielten,

auf Grund des Versuches von Smetánka, welcher an einer Versuchsperson im nüchternen Zustande mit einmaliger Nahrungsaufnahme nach beinahe 2 Monaten unter gleichen Versuchsbedingungen denselben Harnsäurewert vorfand,

auf Grund meiner eigenen Versuche, welche an einem und demselben Subjekte im nüchternen Zustande und unter sonst gleichen Verhältnissen nach einem Intervall von fünfundzwanzig Jahren genau denselben Harnsäurewert konstatierten, und welche dasselbe Resultat an einer anderen Versuchsperson im nüchternen Zustande unter einmaliger purinfreier Nahrungsaufnahme und sonst gleichen Bedingungen nach einem Zeitraume von dreiundeinhalb Jabren lieferten, erkläre ich die Menge der durch einen erwachsenen menschlichen Organismus in bestimmten Zeitperioden unter sonst gleichen Verhältnissen ausgeschiedenen Harnsäure für eine physiologische Konstante desselben. 